\title{
Human hsp70 and HPV16 oE7 fusion protein vaccine induces an effective antitumor efficacy
}

\author{
JINBAO ZONG ${ }^{1}$, CHANGYUAN WANG $^{2}$, BIN LIU $^{3}$, MINGJUN LIU $^{1}$, \\ YONGXIAN CAO $^{1}$, XIUFANG SUN ${ }^{1}$, YUAN YAO $^{1}$ and GUIRONG SUN ${ }^{1}$ \\ ${ }^{1}$ Clinical Laboratory, The Affiliated Hospital of Medical College, Qingdao University, Qingdao 266003; \\ ${ }^{2}$ Department of Dermatology, Qingdao Municipal Hospital, Qingdao 266001; ${ }^{3}$ Department of Rheumatology, \\ The Affiliated Hospital of Medical College, Qingdao University, Qingdao 266003, P.R. China
}

Received January 11, 2013; Accepted April 4, 2013

DOI: $10.3892 / o r .2013 .2445$

\begin{abstract}
The persistent infection by human papilloma virus (HPV) is considered to be the major risk factor of cervical cancer, which is one of the most common cancers in women worldwide. Millions of women are currently infected with high-risk HPV. Thus, it is urgent to develop therapeutic vaccines to eliminate established infection or HPV-related diseases. In the present study, we constructed a very promising therapeutic HPV16 protein vaccine of optimized E7 (oE7)/huhsp70 using human hsp70 linked to HPV16 oE7. Our results demonstrated that vaccination with the oE7/huhsp70 protein vaccine induced a very strong E7-specific $\mathrm{CD} 8^{+} \mathrm{T}$ cell immune response and resulted in a significant therapeutic effect against E7-expressing tumor cells. Our study verifies that huhsp70 is an effective immune adjuvant in the development of tumor therapeutic protein vaccines, and emphasizes that homologous huhsp70 is a promising tool in future human clinical applications.
\end{abstract}

\section{Introduction}

Cervical cancer is a malignant neoplasm arising from cells originating in the cervix uteri. The development of cervical cancer are closely associated with constitutive infection of HPV viruses, of which HPV16 is the most common high-

Correspondence to: Dr Guirong Sun, Clinical Laboratory, The Affiliated Hospital of Medical College, Qingdao University, 16 Jiangsu Road, Qingdao 266003, P.R. China

E-mail: sunguirong@hotmail.com

Abbreviations: HPV, human papilloma virus; $\mathrm{MHC}$, major histocompatibility complex; hsp70, heat shock protein 70; huhsp70, human hsp70; APC, antigen-presenting cell; DC, dendritic cell; oE7, optimized HPV16 E7; TLR, toll-like receptor; LOX-1, Lectin-like, oxidized low-density lipoprotein receptor-1; pRB, retinoblastoma protein

Key words: cervical cancer, protein vaccine, human papilloma virus $16, \mathrm{E} 7$ protein, human heat shock protein 70 risk type, accounting for more than half $(56 \%)$ of all cervical cancers (1). It is well known that Harald zur Hausen, the German researcher who discovered the human papillomavirus, was awarded Noble Prize in Medicine on 2008. The finding of HPV makes prevention and treatment of cervical cancer possible. In addition to preventive vaccines, such as Gardasil and Cervarix (2,3), laboratory researches are paying attention to the treatment of cervical cancer that has been developed. Preventive vaccines do not cause therapeutic effects on existing lesions, thus, it is urgent to focus on the treatment of pre-existing lesions. In addition to traditional surgery, chemotherapy and radiotherapy, therapeutic HPV vaccines are attracting our attention. HPV E7 oncoprotein represent an ideal target for therapeutic intervention because of its constitutive expression in HPV-associated tumors and their crucial role in the induction and maintenance of HPV-associated diseases $(4,5)$.

Versatile therapeutic strategies have been developed (6-10). Various therapeutic HPV vaccines are aiming to interact with professional antigen-presenting cell (APC) such as dendritic cell (DC) to generate specific cell-mediated immunity for clearance of infection and control of HPV-associated cancers $(11,12)$. Protein-based therapeutic HPV vaccines are vaccine candidates commonly tested in clinical trials, compared to other forms of therapeutic HPV vaccines (13). Protein vaccines have some advantages in vaccine development. For example, compared to live-vector based vaccines they are safe. Protein vaccines contain all possible peptide epitopes interacting with the MHC I and II epitopes, so they are not limited by the specificity of MHC. However, protein vaccines are troubled by their low immunogenicity. To overcome this problem and to enhance their potency, we design two novel new strategies, one is optimization of E7 protein by adding MHC-I epitope at $\mathrm{C}$ terminal and MHC-II epitope at $\mathrm{N}$ terminal of fusion protein, and another is the addition of human hsp70 (huhsp70) adjuvant.

Effective antigen process and presentation is the indispensable condition to induce a strong and specific $\mathrm{CD}^{+} \mathrm{T}$ cell response. Because the normal capacity of APC to cross-present antigen is generally low, there is significant interest to develop strategies that enhance the targeting of exogenous antigens to the cross-presentation pathway $(14,15)$. Critical factors that 
determine the efficacy of a vaccine are the amount of delivered antigen and the surroundings in which the antigen is presented to the T cells. The addition of MHC I epitope at $\mathrm{C}$ terminal and MHC II epitope at $\mathrm{N}$ terminal of fusion protein, makes more specific antigen peptides to act with MHC molecules, so that it enlarges the rate of recognition for MHC molecules. Thus, the delivery of more antigens to the MHC class I cross-presentation pathway is one of the keys to the improved capacity of APCs to activate antigen-specific $\mathrm{T}$ cells.

Another strategy is the use of hsp70 to enhance the antigen cross presentation. As an intracellular protein, hsp70 has been shown to play multiple roles in protein folding, transport, and degradation act as molecular chaperones (16). However, it is reported that hsps also are involved in the immune activation in that they transfer their chaperoned protein-cargo to APC for cross-presentation as an extracellular protein released when necrotic cells or secretion in response to cellular stress. Such antigenic cross-presentation is now considered to be a very important process in the action of hsp70 (17-20). The extracellular hsp70 is considered to mediate stimulation of DCs to secrete proinflammatory cytokines and express costimulatory molecules, thus creating the immunogenic environment required for the induction of adaptive $\mathrm{CD}^{+} \mathrm{T}$ cell responses. Several endocytic receptors, such as CD91 and LOX1 have been identified to take part in hsp70-mediated cross-presentation $(21,22)$. When hsp70 binds to its corresponding receptors of CD40, TLR-2 and TLR-4 on the DC, enters the cell plasma and induces activation and maturation DC (23-25). Hsp70 not only activates and regulates innate immunity but also the adaptive immunity. The above attributes of hsp70 suggest its rational use in immunotherapeutic strategies for the treatment of cancer. Thus, this activity of hsp70 is desirable for therapeutic vaccine development.

Our previous study on HPV16 E7/hsp70 DNA vaccine has shown that huhsp70 enhanced more effective antitumor efficacy than mycobacterium tuberculosis hsp70 (26). In this protein vaccine study, we optimized the E7 protein by sitedirected mutagenesis to eradiate the transformation activity, MHC epitope and human hsp70 adjuvant were added to strengthen the cross-presentation potential, and developed the fusion protein of oE7/huhsp70, expecting to produce enhanced antitumor efficacy.

\section{Materials and methods}

Mice and tumor cell line. Female C57BL/6 mice of 6-8-weekold were maintained in the Animal Facility of the Laboratory Animal Center, the Affiliated Hospital of Medical College of Qingdao University. All animal procedures were performed according to the approved protocols and in accordance with recommendations for the proper use and care of laboratory animals. Care was taken to minimize pain and discomfort to all animals during the procedures in this study. TC-1 cells purchased from Shanghai Meilian Biotechnology Co., Ltd., (Shanghai, China) were primary pulmonary epithelial cells of C57BL/6 mice co-transformed with HPV16 E6, E7 and activated c-Ha-ras oncogenes. The cells were grown in RPMI-1640 supplemented with $10 \%$ (v/v) fetal bovine serum, $50 \mathrm{U} / \mathrm{ml}$ penicillin/streptomycin, $2 \mathrm{mM}$ L-glutamine,
$1 \mathrm{mM}$ sodium pyruvate, $2 \mathrm{mM}$ non-essential amino acids and $0.4 \mathrm{mg} / \mathrm{ml} \mathrm{G} 418$ at $37^{\circ} \mathrm{C}$ with $5 \% \mathrm{CO}_{2}$.

Plasmids construction. HPV16 oE7 gene was synthesized by Shanghai Generay Biotech Co., Ltd. (Shanghai, China) and cloned into pMD18T vector by using two primers, forward primer 5'-GCCATATGATGGACAGCTCAGAGGAGG-3' $(N d e \mathrm{I})$ and reverse primer 5'-CGGGATCCGGTTACAATA TTGTAATG-3' (BamHI), then was subcloned into pET-30a (+) (Novagen, Darmstadt, Germany) and generated the pET-30a (+)-oE7. The huhsp70 gene (GenBank NM_005345) was amplified by using forward primer 5'-GCGGATCCCATGGC CAAAGCCGCGGC-3' (BamHI) and reverse primer 5'-CGCT CGAGCTAATCTACCTCCTCAATGGTG-3' (XhoI) from pMSHsp70, a gift of Prof. R.I. Morimoto of the Northwestern University (Evanston, IL, USA), then subcloned into pET-30a (+) and generate pET-30a (+)-huhsp70. To generate pET-30a (+)-oE7/ huhsp70, huHSP70 digested with BamHI and XhoI from pET-30a (+)-huhsp70 was ligated into BamHI/XhoI -digested pET-30a (+)-oE7 without the stop code of oE7. All constructs were validated by restriction enzyme digestion and DNA sequencing.

Expression, purification and analysis of oE7, huhsp70 and oE7/huhsp70 proteins. E. coli strain BL21 (DE3) (Novagen) was used as the host bacterial for all recombinant protein production. All transformants were grown in Luria Broth (LB) medium containing $50 \mu \mathrm{g} / \mathrm{ml}$ kanamycin. When OD600 reach about 0.5 , engineered bacterial was induced with $1 \mathrm{mM}$ isopropyl-b-D-thiogalactopyranoside (IPTG) for $4 \mathrm{~h}$ at $37^{\circ} \mathrm{C}$. Cells were harvested by centrifugation at $10,000 \mathrm{x} \mathrm{g}$ for 10 min at $4^{\circ} \mathrm{C}$, the supernatant was discarded and the cells resuspended in $4 \mathrm{ml}$ cold bingding buffer with $0.1 \%$ NP-40. Using the His•Bind ${ }^{\circledR}$ Purification kit (Novagen, No. 70239) to purify these recombinant proteins. The identity and the purity of the recombinant proteins were determined by SDS-PAGE. Concentrations of proteins were measured by the Bradford assay. To confirm the identity of the recombinant protein, all purified recombinant proteins were verified by western blot analysis against the human hsp70 antibody (sc-32239; Santa Cruz Biotechnology, Santa Cruz, CA, USA) and/or HPV16 E7 antibody (sc-51951, Santa Cruz Biotechnology).

ELISPOT assay. BD ELISPOT plates (BD BioSciences) were coated with $5 \mu \mathrm{g} / \mathrm{ml}$ rat anti-mouse IFN- $\gamma$ antibody in $100 \mu \mathrm{l}$ of PBS. After overnight incubation at $4^{\circ} \mathrm{C}$, the wells were washed and blocked with RPMI-1640 culture medium containing $10 \%$ fetal bovine serum. Different concentrations of freshly isolated spleen cells from each vaccinated mouse group, from $1 \times 10^{6}$ to $1.25 \times 10^{5} /$ well, were added to the wells along with $50 \mathrm{IU} / \mathrm{ml}$ IL-2 and $1 \mu \mathrm{g} / \mathrm{ml} \mathrm{E7-specific} \mathrm{MHC} \mathrm{class} \mathrm{I} \mathrm{CD8}{ }^{+} \mathrm{T}$ cell epitope (H-2 Db, amino acids 49-57, RAHYNIVTF). After culture at $37^{\circ} \mathrm{C}$ for $24 \mathrm{~h}$, the plate was washed and then followed by incubation with $2.5 \mu \mathrm{g} / \mathrm{ml}$ biotinylated IFN- $\gamma$ antibodies in $100 \mu \mathrm{l}$ in PBS containing $10 \% \mathrm{FCS}$ at $4^{\circ} \mathrm{C}$ overnight. After washing, avidin-HRP in $100 \mu \mathrm{l}$ of PBS was added and incubated for $1 \mathrm{~h}$ at room temperature. After washing five times, spots were developed by addition of $100 \mu 1$ AEC solution. The spots were counted using an ELISPOT Reader system. 
Flow cytometry analysis to detect IFN- $\gamma$ secretion by E7-specific $\mathrm{CD}^{+} \mathrm{T}$ cells. To detect E7-specific CD8 ${ }^{+} \mathrm{T}$-cell responses, splenocytes from vaccinated groups of mice were incubated with the MHC class I E7 peptide. Golgistop (BD BioSciences) was added $6 \mathrm{~h}$ before harvesting the cells from the culture. The cells were then washed once in staining buffer and labeled with FITC-conjugated rat anti-mouse CD8 antibodies. The cells were fixed and permeabilized using a Cytofix/Cytoperm kit (BD BioSciences) according to the manufacturer's instructions and then were stained for PE-conjugated anti-IFN- $\gamma$. FITC-conjugated rat $\operatorname{IgG} 2_{\mathrm{a}, \mathrm{k}}$ or $\mathrm{PE}$-conjugated rat $\mathrm{IgG}_{1}$ isotype control antibody were all purchased from BD BioSciences. Analyses were performed on a Beckman Coulter Epics XL (Beckman Coulter Inc., Brea, CA, USA).

Cytotoxicity assays. C57BL/6 mice were immunized intramuscularly (i.m.) with PBS, 2 nmol oE7, huhsp70 and oE7/hsp70 at the M. quadriceps, respectively. These injections were repeated after 1 week. Two weeks after the last booster, $1 \times 10^{6}$ splenocytes were cocultured with $5 \times 10^{4}$ irradiated TC-1 helper cells in RPMI-1640 supplemented with 10\% FCS and $20 \mathrm{U} / \mathrm{ml} \mathrm{IL}-2$ at $37^{\circ} \mathrm{C}$ in $5 \% \mathrm{CO}_{2}$. After 3 days of stimulation, TC-1 target cells were plated at $1 \times 10^{4}$ cells/well on 96 -well U-bottomed plates (Costar), then the prepared splenocytes (effector cells) were added in a final volume of $100 \mu \mathrm{l}$ at 40:1, 20:1, 10:1 and 5:1 ratio, respectively. The CytoTox 96 NonRadioacive Cytotoxicity Assay kit (Promega Inc.) was used to determine the cytotoxic activity of the effector cells against TC-1 tumor cells according to the manufacturer's protocol. The percentage of cytotoxicity was calculated by the formula: [A (Experimental) - A (Effector spontaneous) - A (Target spontaneous)] x 100/[A (Target maximum - Target spontaneous)].

Anti-E7 ELISA. The anti-HPV16 E7 antibodies in the sera of vaccinated mice were identified by ELISA. Each well of a 96-microwell plate was coated with $100 \mu \mathrm{l}$ of $1 \mu \mathrm{g} / \mathrm{ml}$ purified HPV16 oE7 proteins and incubated at $4^{\circ} \mathrm{C}$ overnight. The wells were then blocked with PBS containing 5\% BSA. Two weeks after the last booster, sera were prepared from the mice, serially diluted in PBS, added to the ELISA wells and incubated for $2 \mathrm{~h}$. After washing with PBS-T containing $0.05 \%$ Tween-20, the plate was incubated with 1:3,000 dilution of an HRP-conjugated goat anti-mouse IgG antibody (sc-2005; Santa Cruz Biotechnology) at room temperature for $1 \mathrm{~h}$. The plate was washed three times, tetramethyl-benzidine substrate was added and incubated away from light at $37^{\circ} \mathrm{C}$ for $15 \mathrm{~min}$. The reaction was stopped with $50 \mu \mathrm{l}$ of $2 \mathrm{M} \mathrm{H}_{2} \mathrm{SO}_{4}$. The ELISA plate was read with a standard ELISA reader at $450 \mathrm{~nm}$.

In vivo tumor treatment experiments. To test the ability of protein vaccination to inhibit the growth of established tumors, C57BL/6 mice (five/group) were subcutaneously (s.c.) challenged with $7.5 \times 10^{4} \mathrm{TC}-1$ cells per mouse in the right flank. Three days after the challenge with TC-1 cells, mice were given $200 \mu \mathrm{l}$ of PBS, $2 \mathrm{nmol} \mathrm{oE} 7$, huhsp70 or oE7/huhsp70. One week later, these mice were strengthened with the same regimen as the first vaccination. Mice were monitored twice a week for tumor growth.

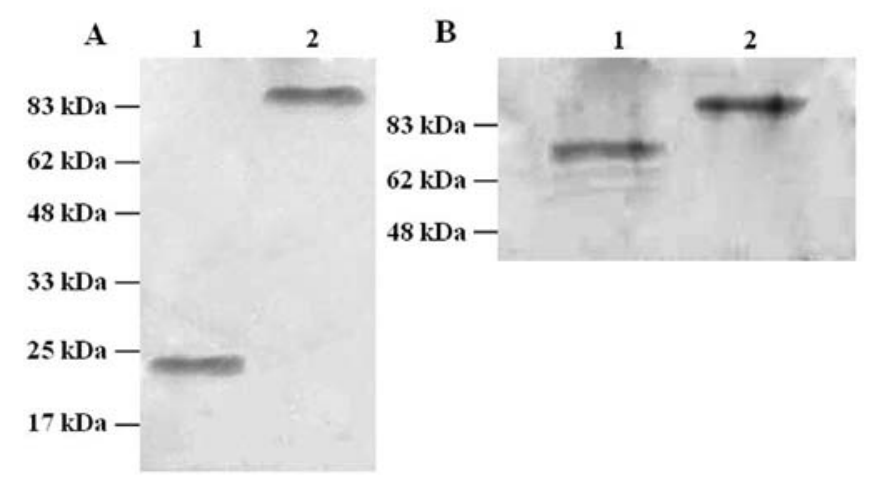

Figure 1. Analysis of oE7, huhsp70 oE7/huhsp70 proteins by western blot analysis. (A) Analysis of purified oE7 protein (lane 1) and oE7/huhsp70 protein (lane 2) by using human HPV16 E7 antibody. (B) Analysis of purified huhsp70 protein (lane 1) and oE7/huhsp70 protein (lane 2) by using human hsp70 antibody.

Statistical analysis. The mean of two sample comparison of Poisson distribution was used to analyze ELISPOT and FACS data. ELISA with the Student's t-test, cytotoxicity assays and tumor treatment data were analyzed by the Fisher's exact probabilities in a $2 \times 2$ table. Differences were considered statistically significant at $\mathrm{P}<0.01$.

\section{Results}

Production and analysis of recombinant proteins.oE7, huhsp70 and oE7/huhsp70 recombinant proteins were expressed effectively in E. coli BL21 in a form of inclusion body. All proteins with his-tag were purified by the His $\bullet$ Bind ${ }^{\circledR}$ Purification kit. All recombinant proteins were purified to $>90 \%$. The recombinant proteins used in this study contained $<0.05$ endotoxin units (EU) $/ \mu \mathrm{g}$ as measured by the chromogenic Limulus amebocyte lysate assay. Concentrations of proteins were measured by the Bradford assay. The expression and analysis of recombinant proteins were confirmed by western blot analysis (Fig. 1).

oE7/huhsp70 fusion protein significantly induces strong E7-specific $C D 8^{+} T$ cell responses. $\mathrm{CD}^{+} \mathrm{T}$ cells are the most important cells involved in antitumor effect, while the ELISPOT assays and flow cytometry analysis are the two representative methods. To evaluate whether oE7/huhsp70 is able to prime strong E7-specific CD8 ${ }^{+} \mathrm{T}$ cells, oE7 or huhsp70, the mice were immunized i.m. with $200 \mu \mathrm{l}$ of PBS, 2 nmol oE7, huhsp70 or oE7/huhsp70, which was repeated after 1 week. Two weeks after the second immunization, splenocytes were harvested and stimulated with $1 \mu \mathrm{g} / \mathrm{ml} \mathrm{H}-2 \mathrm{Db}$-restricted E7 peptide. In ELISPOT assays, as shown in Fig. 2, vaccination with oE7/huhsp70 generated significantly higher percentage of E7-specific IFN- $\gamma$-secreting $\mathrm{CD}^{+} \mathrm{T}$ cell precursors than PBS, oE7 or huhsp70. Similar results were also obtained by intracellular IFN- $\gamma$ staining with flow cytometry analysis. As shown in Fig. 3, vaccination with oE7/huhsp70 also generated the highest percentage of E7-specific IFN- $\gamma$-secreting CD8 ${ }^{+}$ $\mathrm{T}$ cell precursors compared to the others. In accordance with the ELISPOT and flow cytometry analysis, cytotoxicity assays showed that splenocytes of the mice immunized with oE7/ 


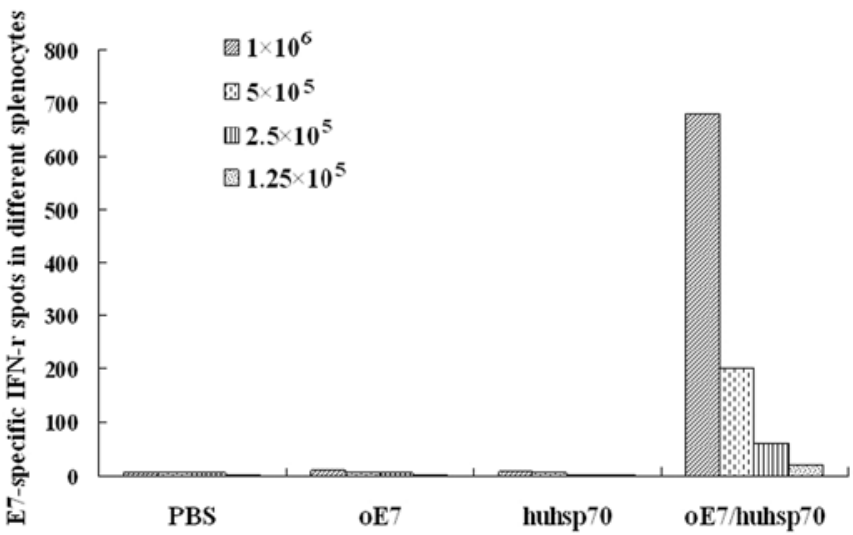

Figure 2. The E7-specific IFN- $\gamma$ spot numbers were the mean of triplicates \pm $\mathrm{SE}$ in each vaccinated group at different cell numbers. Results shown here are for E7-specific spot-forming cells from four groups and from one representative experiment of three performed tests.

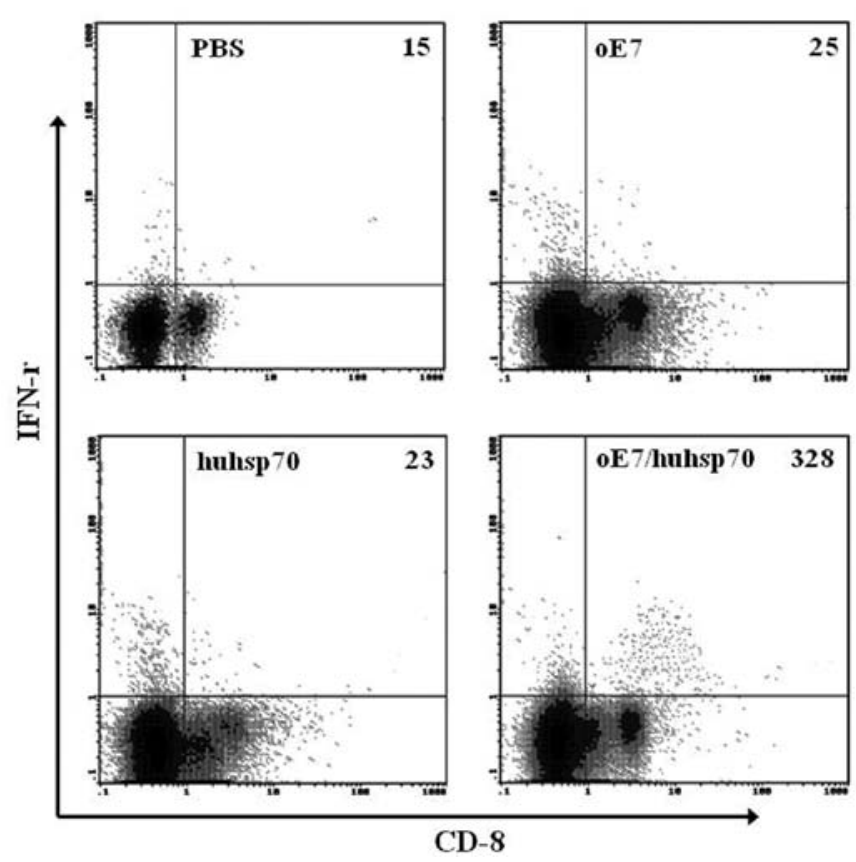

Figure 3. Analysis of IFN- $\gamma$-secreting E7-specific $\mathrm{CD} 8^{+} \mathrm{T}$ cells with flow cytometry. Splenocytes from vaccinated mice were cultured in vitro with the E7 peptide (amino acids 49-57) overnight and stained for both CD8 and intracellular IFN- $\gamma$. Mice vaccinated with $\mathrm{oE7} / \mathrm{huhsp} 70$ protein generated the highest IFN- $\gamma^{+} / \mathrm{CD}^{+}$double-positive $\mathrm{T}$ cells. The number of double-positive $\mathrm{T}$ cells in $1 \times 10^{5}$ splenocytes is indicated in the upper right corner. Results shown here are from one representative experiment of three performed tests.

huhsp70 were more cytotoxic to TC-1 target cells expressing E7 than those in the mice immunized with PBS, oE7 or huhsp70 $(\mathrm{P}<0.01)($ Fig. 4). Taken together, our results suggest that $\mathrm{oE} 7 /$ huhsp70 fusion protein vaccine generates the highest E7-specific $\mathrm{CD}^{+} \mathrm{T}$ cell response compared with the other vaccinated groups. Results shown here are all representative of three repeated experiments.

Vaccination with oE7/huhsp70 induces an E7-specific antibody response. The quantity of anti-HPV16 E7 antibodies in the sera of the vaccinated mice was determined by ELISA

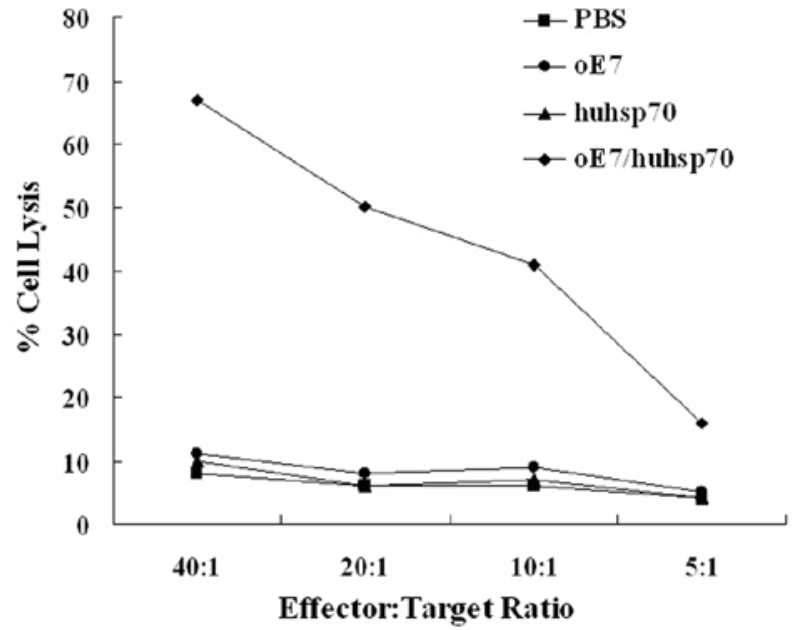

Figure 4. E7-specific lysis against TC-1 cells by $\mathrm{CD} 8^{+} \mathrm{T}$ cells induced by vaccination with various proteins. Mice were vaccinated as described in the method of cytotoxicity assays. The splenocytes of mice were harvested and restimulated with irradiated TC-1 cells. The percentage of specific lysis of $\mathrm{CD} 8^{+} \mathrm{T}$ cells on $\mathrm{TC}-1$ target cells was determined by cytotoxicity assays. The E7-specific lysis of $\mathrm{CD}^{+} \mathrm{T}$ from mice vaccinated with oE7/huhsp70 was higher than those vaccinated with PBS, oE7 or huhsp70 $(\mathrm{P}<0.01, \mathrm{n}=5)$.

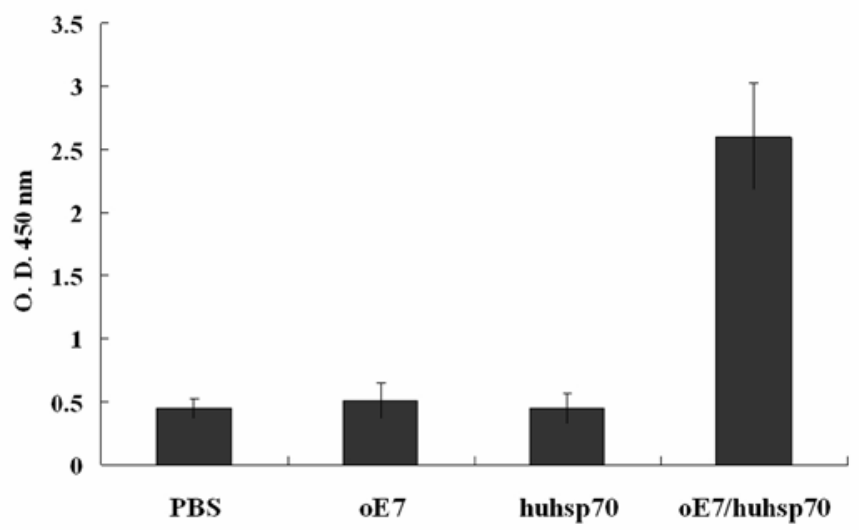

Figure 5. E7-specific antibody responses in vaccinated mice. C57BL/6 mice were immunized with PBS control and serum samples were obtained from immunized mice 2 weeks after vaccination. The presence of the E7-specific antibody was detected by ELISA using serial dilution of sera. The results from the 1:10 dilution are presented showing the mean absorbance $(\mathrm{A} 450 \mathrm{~nm}) \pm \mathrm{SE}$.

2 weeks after the last vaccination. As shown in Fig. 5, only mice vaccinated with $\mathrm{oE} 7 /$ huhsp70 fusion protein produce a robust antibody response $(\mathrm{P}<0.01)$, indicating that secreted oE7/huhsp70 stimulates B-cell responses.

Tumor treatment experiments. In order to examine the E7-specific $\mathrm{CD}^{+} \mathrm{T}$ response elicited by oE7/huhsp70 fusion protein further, we investigated the efficacy of each vaccine to induce the eradication of pre-existing TC-1 tumor expressing E7 in vivo. Female C57BL/6 mice were challenged with $7.5 \times 10^{4} \mathrm{TC}-1$ cells per mouse s.c. in the right flank. Three days after the challenge with TC-1 cells, mice were given $200 \mu \mathrm{l}$ of PBS, $2 \mathrm{nmol} \mathrm{oE7,} \mathrm{huhsp70} \mathrm{or} \mathrm{oE7/}$ huhsp70. One week later, these mice were boosted with the same procedure as the first vaccination. As shown in Fig. 6, $100 \%$ of the mice immunized with oE7/huhsp70 were tumor- 


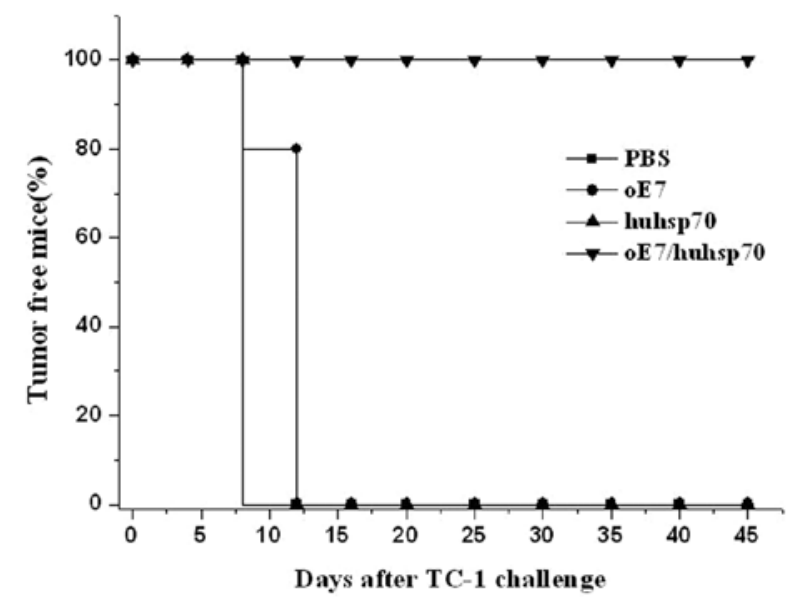

Figure 6. In vivo tumor treatment experiments. Vaccination with oE7/huhsp70 protein enhanced the antitumor immunity more significantly than PBS oE7 and huhsp70. Vaccination with oE7/huhsp70 protein eradicated preimplanting TC-1 tumor cells, $100 \%$ of mice were tumor-free until day 45 after TC-1 challenge, while $100 \%$ of mice vaccinated with PBS, huhsp70 form a tumor at the 12th day and maintain the tumor until day 45 except the mice vaccinated with oE7 forming a tumor at day 16. Each mouse was initially challenged s.c. with $7.5 \times 10^{4} \mathrm{TC}-1$ cells in the right flank and then was vaccinated i.m. with PBS, oE7, huhsp70 and oE7/huhsp70 on days 3 and 10 The mice were monitored for tumor growth twice a week, each group comprised five mice. The results shown here are from a representative experiment of two performed.

free until day 45. In contrast, mice receiving PBS or huhsp70 developed tumor growth on the 12th day until the 45th day, while 4 mice receiving oE7 developed tumor growth on the 12th day and 1 mouse developed tumor growth on the 16th day until the 45 th day. There was a statistically significant difference in the therapeutic effect of TC-1 tumor between oE7/huhsp70 and other vaccinated group $(\mathrm{P}<0.01)$. These results show that $\mathrm{oE} 7 /$ huhsp70 fusion protein vaccine is the most potent and promising therapeutic vaccine against E7-expressing tumors among the various protein vaccines which we have tested.

\section{Discussion}

Results of the present study revealed that fusion protein of oE7/huhsp70 not only stimulated very strong CD8 ${ }^{+}$ T-cell responses but also induced an effective E7 antibody response, indicating that $\mathrm{E} 7$-specific $\mathrm{CD}^{+} \mathrm{T}$-cell responses and $\mathrm{B}$ cell mediated humoral immunity play important roles in anti-tumor effect with this protein vaccine, $\mathrm{T}$ helper cell responses might be simultaneously produced. The previously reported increased HPV-16 E7-specific IgG levels appeared to be correlated with a positive therapeutic effect (27). An important condition of tumor adaptive immunity is MHC I and II presentation of self and nonself peptides to $\mathrm{CD}^{+}$ $\mathrm{T}$ and $\mathrm{CD}^{+} \mathrm{T}$ cells respectively. Endogenous antigens are generally presented by MHC I, and exogenous antigens by MHC II. In the present study, the addition of MHC-I epitope at $\mathrm{C}$ terminal and $\mathrm{MHC}-\mathrm{II}$ epitope at $\mathrm{N}$ terminal of fusion protein, aimed to enhance the quantity of epitope, increased the chance of involvement of antigen presentation, and strengthened the E7-specific $\mathrm{CD}^{+} \mathrm{T}$-cell responses and E7 antibody response.
It is well known that exogenous antigens can also be internalized and displayed by MHC I molecules through a widely accepted mechanism, called as cross-priming or cross-presentation. Many studies have recognized and confirmed that hsp70 is involved in the antigen cross-priming and induction of effective tumor specific $\mathrm{CD} 8^{+} \mathrm{T}$-cell responses $(19,20,28)$. It has been shown that pathogen-derived molecules are danger signals and are able to activate innate immunity that in turn regulates and influences development of adaptive immunity (29,30). For example, mycobacterium tuberculosis heat shock protein 70 has been shown to exert a potent adjuvant effect in therapeutic vaccination against tumors (31). These studies suggest that mycobacterium tuberculosis also act as danger signal aside from the molecular chaperon and cross-presentation function. However, it is clear that there is no shortage of controversy on the action mechanism of the different species originated hsp70 in tumor therapeutic vaccines. Bendz et al (15) have shown that huhsp70 enhances tumor antigen presentation through complex formation and intracellular antigen delivery without innate immune signaling, in addition, the fusion DNA vaccine of mouse hsp70 with HPV16 E7 showed that autologous hsp70 was highly potent in enhancing antigen-specific immune responses (32). These previous therapeutic HPV vaccine reports showed that whether mouse or human hsp70, especially huhsp70 induced very strong effective $\mathrm{CD}^{+} \mathrm{T}$-cell responses and antitumor effect, even $100 \%$ of mice were tumor-free until day 60 after in vivo TC-1 challenge in tumor protection and treatment experiments (26), which indicated that hsp70 plays an important role in tumor therapeutic vaccine not for its danger signal alerting ability but for its molecular chaperon and cross-presentation function. Thus, huhsp70 fulfills these central requirements of a tumor vaccination tool and a strong immune adjuvant.

Safety also is a critical factor to be considered during development of effective vaccines. The safety problem of $\mathrm{oE} 7 /$ huhsp70 fusion protein vaccine originats from two areas, one is the potentially malignant transformation activity of E7 protein, and another is the homology of hsp70 adjuvant itself. E7 protein has a tendency to bind with the tumor suppressor protein $\mathrm{pRB}$ of the host, resulting in chromosomal aberration and finally development of malignant transformation of the host cells. In order to eradiate the transformation activity of E7 and enhance its immunogenicity, we muted two zinc finger binding areas and the pRB binding area of E7. Hsp70 are highly conserved from prokaryotic to eukaryotic organisms not only functionally but also structurally. However, allogenous hsp might induce immune reaction to interrupt the recognition of antigen and antigen presenting cells in mouse or human (33), or trigger the induction and expansion of regulatory T-cells with immunosuppressive functions $(34,35)$. A potential safety concern arises from the existence of T-lymphocytes cross-reactive with mycobacterial and human hsp (36). Although we cannot discount this risk entirely, we believe that in a clinical setting the use of a human protein will reduce the risk of autoimmunity due to cross-reactive T-lymphocytes primed by the hsp70 vaccines. Mycobacterium tuberculosis, mouse and human hsp70 can induce effective antitumor effects, being a self-protein, toxicity issues are unlikely to occur, taking into account the future clinical test safety, we recommend self huhsp70 as a 
potential and promising candidate adjuvant to help the therapeutic vaccine to produce enhanced antitumor effects.

In summary, huhsp70 may be a useful and promising antigen delivery vehicle for a wide variety of antigens, crosspresentation is applicable for APC and in various settings of immune modulation. Our findings not only provide novel insights into the mechanism by which huhsp70 stimulates $\mathrm{T}$ cell responses but also provide reference for the development of highly effective molecular tools and immune adjuvant in other tumor biological treatments.

\section{Acknowledgements}

We gratefully acknowledge Professor Richard I. Morimoto (Northwest University, Evanston, IL, USA) for kindly providing the pMSHsp70 plasmid and Professor Xuemei Xu (Chinese Academy of Medical Sciences and Peking Union Medical College, Beijing, China) for providing the PVR1012mE7/HuHSP70 plasmid. The present study was supported by a grant from the Nature Science Foundation for Young Scholars of Shandong Province (ZR2010HQ009).

\section{References}

1. Clifford GM, Smith JS, Plummer M, et al: Human papillomavirus types in invasive cervical cancer worldwide: a meta-analysis. $\mathrm{Br}$ J Cancer 88: 63-73, 2003.

2. Siddiqui MA and Perry CM: Human papillomavirus quadrivalent (types $6,11,16,18)$ recombinant vaccine (Gardasil). Drugs 66: 1263-1273, 2006.

3. Crosbie EJ and Kitchener HC: Cervarix ${ }^{\mathrm{TM}}$-a bivalent L1 viruslike particle vaccine for prevention of human papillomavirus type 16- and 18-associated cervical cancer. Expert Opin Biol Ther 7: 391-396, 2007.

4. Dell G and Gaston K: Human papillomaviruses and their role in cervical cancer. Cell Mol Life Sci 58: 1923-1942, 2001.

5. zur Hausen H: Papillomaviruses and cancer: from basic studies to clinical application. Nat Rev Cancer 2: 342-350, 2002.

6. Li YL, Liu J, Liu JN, et al: Immunization of protein HPV16 E7 in fusion with mouse HSP70 inhibits the growth of TC-1 cells in tumor bearing mice. Vaccine 29: 5959-5962, 2011.

7. de Vos van Steenwijk PJ, Ramwadhdoebe TH, Löwik MJ, et al: A placebo-controlled randomized HPV16 synthetic long-peptide vaccination study in women with high-grade cervical squamous intraepithelial lesions. Cancer Immunol Immunother 61: 1485-1492, 2012

8. Zhou L, Zhu T, Ye X, et al: Long-term protection against human papillomavirus e7-positive tumor by a single vaccination of adeno-associated virus vectors encoding a fusion protein of inactivated e7 of human papillomavirus 16/18 and heat shock protein 70. Hum Gene Ther 21: 109-119, 2010.

9. Peng S, Monie A, Pang X, et al: Vascular disrupting agent DMXAA enhances the antitumor effects generated by therapeutic HPV DNA vaccines. J Biomed Sci 8: 18-21, 2011.

10. Santin AD, Hermonat PL, Ravaggi A, et al: Induction of human papillomavirus-specific CD4 and CD8 lymphocytes by E7-pulsed autologous dendritic cells in patients with human papillomavirus type 16-and 18-positive cervical cancer. J Virol 73: 5402-5410, 1999.

11. Kim D, Hoory T, Wu TC, et al: Enhancing DNA vaccine potency by combining a strategy to prolong dendritic cell life and intracellular targeting strategies with a strategy to boost $\mathrm{CD} 4^{+} \mathrm{T}$ cell Hum Gene Ther 18: 1129-1139, 2007.

12. Hauser H and Chen SY: Augmentation of DNA vaccine potency through secretory heat shock protein-mediated antigen targeting. Methods 31: 225-231, 2003.

13. $\mathrm{Ma} \mathrm{B}, \mathrm{Xu} \mathrm{YJ}$, Hung $\mathrm{CF}$, et al: $\mathrm{HPV}$ and therapeutic vaccines: Where are we in 2010? Curr Cancer Ther Rev 6: 81-103, 2010.

14. Maecker HT, Ghanekar SA, Suni MA, et al: Factors affecting the efficiency of $\mathrm{CD}^{+} \mathrm{T}$ cell cross-priming with exogenous antigens. J Immunol 166: 7268-7275, 2001.
15. Bendz H, Ruhland SC, Pandya MJ, et al: Human heat shock protein 70 enhances tumor antigen presentation through complex formation and intracellular antigen delivery without innate immune signaling. J Biol Chem 282: 31688-31702, 2007.

16. Brodsky JL and Chiosis G: Hsp70 molecular chaperones: emerging roles in human disease and identification of small molecule modulators. Curr Top Med Chem 6: 1215-1225, 2006.

17. Calderwood SK, Theriault JR and Gong J: Message in a bottle: role of the $70-\mathrm{kDa}$ heat shock protein family in anti-tumor immunity. Eur J Immunol 35: 2518-2527, 2005.

18. Lancaster GI and Febbraio MA: Exosome-dependent trafficking of HSP70: a novel secretory pathway for cellular stress proteins. J Biol Chem 280: 23349-23355, 2005.

19. Binder RJ and Srivastava PK: Peptides chaperoned by heatshock proteins are a necessary and sufficient source of antigen in the cross-priming of CD8 ${ }^{+} \mathrm{T}$ cells. Nat Immunol 6: 593-599, 2005.

20. Binder RJ, Blachere NE and Srivastava PK: Heat shock proteinchaperoned peptides but not free peptides introduced into the cytosol are presented efficiently by major histocompatibility complex I molecules. J Biol Chem 276: 17163-17171, 2001.

21. Tobian AA, Canaday DH, Boom WH, et al: Bacterial heat shock proteins promote CD91-dependent class I MHC crosspresentation of chaperoned peptide to $\mathrm{CD}^{+} \mathrm{T}$ cells by cytosolic mechanisms in dendritic cells versus vacuolar mechanisms in macrophages. J Immunol 172: 5277-5286, 2004.

22. Delneste Y, Magistrelli G, Gauchat J, et al: Involvement of LOX-1 in dendritic cell-mediated antigen cross-presentation. Immunity 17: 353-362, 2002.

23. Asea A, Kraeft SK, Kurt-Jones EA, et al: HSP70 stimulates cytokine production through a CD14-dependent pathway, demonstrating its dual role as a chaperone and cytokine. Nat Med 6: 435-442, 2000.

24. Singh-Jasuja H, Scherer HU, Hilf N, et al: The heat shock protein gp96 induces maturation of dendritic cells and down-regulation of its receptor. Eur J Immunol 30: 2211-2215, 2000.

25. Kuppner MC, Gastpar R, Gelwer S, et al: The role of heat shock protein (hsp70) in dendritic cell maturation: hsp70 induces the maturation of immature dendritic cells but reduces DC differentiation from monocyte precursors. Eur J Immunol 31: 1602-1609, 2001.

26. Zong JB, Peng QL, Wang QY, et al: Human HSP70 and modified HPV16 E7 fusion DNA vaccine induces enhanced specific CD8 ${ }^{+}$ T cell responses and anti-tumor effects. Oncol Rep 22: 953-961, 2009.

27. Van Doorslaer K, Reimers LL, Studentsov YY, et al: Serological response to an HPV16 E7 based therapeutic vaccine in women with high-grade cervical dysplasia. Gynecol Oncol 116: 208-212, 2010.

28. Srivastava P: Roles of heat-shock proteins in innate and adaptive immunity. Nat Rev Immunol 2: 185-194, 2002.

29. Stewart GR and Young DB: Heat-shock proteins and the hostpathogen interaction during bacterial infection. Curr Opin Immunol 16: 506-510, 2004.

30. Segal BH, Wang XY, Dennis CG, et al: Heat shock proteins as vaccine adjuvants in infections and cancer. Drug Discov Today 11: 534-540, 2006

31. Harmala LA, Ingulli EG, Curtsinger JM, et al: The adjuvant effects of Mycobacterium tuberculosis heat shock protein 70 result from the rapid and prolonged activation of antigen-specific $\mathrm{CD}^{+} \mathrm{T}$ cells in vivo. J Immunol 169: 5622-5629, 2002.

32. Li Y, Subjeck J, Yang G, et al: Generation of anti-tumor immunity using mammalian heat shock protein 70 DNA vaccines for cancer immunotherapy. Vaccine 24: 5360-5370, 2006.

33. Ma JH, Sui YF, Ye J, et al: Heat shock protein 70/MAGE-3 fusion protein vaccine can enhance cellular and humoral immune responses to MAGE-3 in vivo. Cancer Immunol Immunother 54: 907-914, 2005.

34. van Eden W, Koets A, van Kooten P, et al: Immunopotentiating heat shock proteins: negotiators between innate danger and control of autoimmunity. Vaccine 21: 897-901, 2003.

35. Rook GA, Martinelli R and Brunet LR: Innate immune responses to mycobacteria and the downregulation of atopic responses. Curr Opin Allergy Clin Immunol 3: 337-342, 2003.

36. Salvetti M, Ristori G, Buttinelli C, et al: The immune response to mycobacterial $70-\mathrm{kDa}$ heat shock proteins frequently involves autoreactive $\mathrm{T}$ cells and is quantitatively disregulated in multiple sclerosis. J Neuroimmunol 65: 143-153, 1996. 